\title{
Copper Ion Sensing
}

\author{
Ryo KaTO \\ Cooperative Research Facility Center, Toyohashi University of Technology, 1-1 Hibarigaoka, Tenpaku-cho, \\ Toyohashi, Aichi 441-8580, Japan
}

Copper has been used by human beings for a long time in addition to iron and gold. Humans have used copper for various reasons such as in bronze weapons, rituals, agricultural tools, manufacturing of regenerated fibers (copper ammonia rayon), agricultural chemicals, and power transmission lines. It is well known that copper is an essential trace element in organisms; however, excess copper ions can exhibit adverse effects. Therefore, considerable attention has been paid to monitor copper ion concentrations in environment. In the field of analytical chemistry, there are numerous reports regarding the development of analytical tools for copper ions aimed at monitoring the concentrations of copper ions in wastewater from factories, in the environment, and in living tissues.

ICP-AES, ICP-MS, and atomic absorption spectroscopy (AAS) are mainstream methods for analyzing metal ions including copper. At present, the development of analytical reagents that form complexes with copper ions as well as absorption or fluorescence techniques for the purpose of convenient copper ion detection and applications in bioimaging have been actively studied. ${ }^{1,2}$ Fei and co-workers developed a porphyrin-based chemosensor, ${ }^{3}$ Lan and co-workers developed a rhodamine-based chemosensor for copper and iron, ${ }^{4}$ and Yang and co-workers developed an oligo peptide chemosensor for copper ions. ${ }^{5}$ These sensors exhibit changes in absorption or fluorescence upon coordination to copper ions. Recently, a new type of analytical reagent using surface modified nanoparticles (NP) was also reported. ${ }^{6,7}$ Satheeshkumar and co-workers

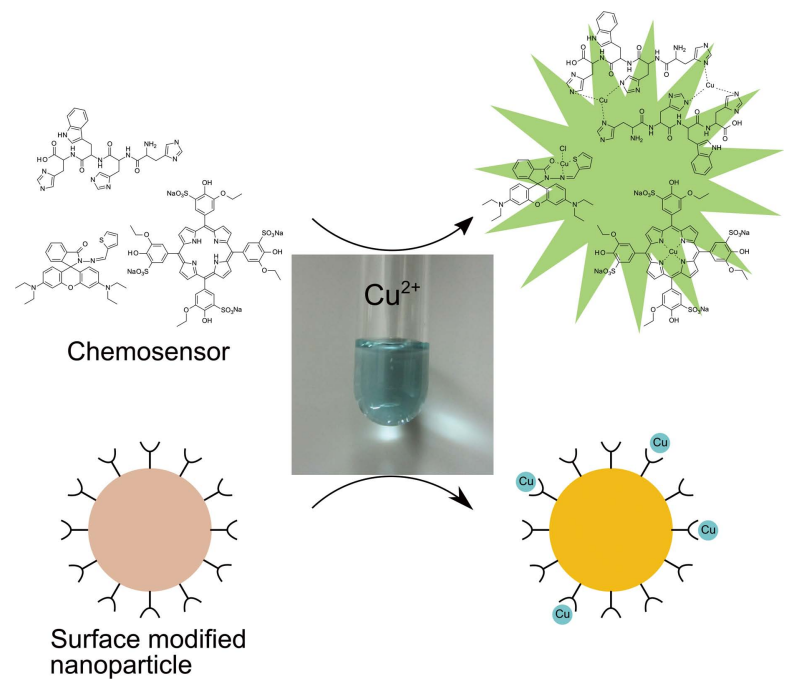

Schematic image of copper ion sensing.

E-mail: rk001@edu.imc.tut.ac.jp developed an oxidized tyrosine-modified silver NP, ${ }^{8}$ Wang and co-workers developed a gold core platinum shell NP, ${ }^{9}$ and Kumar and co-workers developed a thiourea-modified $\mathrm{CdS}$ quantum dot. ${ }^{10}$ Zheng and co-workers reported the fluorescence detection of copper ions using hyperbranched polyamidoamine, which is an organic material that is similar to a dendrimer. ${ }^{11}$ Iwai reported the removal of trace levels of copper ions in seawater. ${ }^{12}$

The aforementioned reports involved cases in which copper ions played a role other than as an analyte. Ito and co-workers determined the concentration of nitrilotriacetate and other chelating agents in detergents by titration of copper ions. ${ }^{13} \mathrm{Wu}$ and co-workers used the absorption of copper ions at around $800 \mathrm{~nm}$ (copper aquo complex) as a means of evaluating the performance of microfluidic optical cells. ${ }^{14}$

As described above, copper ions are important metal ions, which are often analyzed for various purposes and by various methods. As such, the importance of copper ions in the field of analytical chemistry will remain unchanged.

Keywords Copper, chemosensor

\section{References}

1. A. Ramdass, V. Sathish, E. Babu, M. Velayudham, P. Thanasekaran, and S. Rajagopal, Coord. Chem. Rev., 2017 , 343, 278.

2. D. Udhayakumari, S. Naha, and S. Velmathi, Anal. Methods, 2017, 9, 552.

3. Q. Fei, H. Shan, Y. Huan, Z. Zhang, H. Mi, H. Xu, G. Li, F. Chen, and G. Feng, Anal. Sci., 2016, 32, 745.

4. T. Lan, F.-H. Wang, X.-J. Xi, C.-W. Cheng, W. Lei, M.-Z. Xia, and F.-Y. Wang, Anal. Sci., 2016, 32, 1223.

5. T. Yang, F. Zhu, T. Zhou, J. Cao, Y. Xie, M. Zhang, Y. Wang, D.-S. Cao, Q. Lin, and L. Zhang, Anal. Sci., 2017, 33, 191.

6. E. Oliveira, C. Nunez, H. M. Santos, J. Fernandez-Lodeiro, A. Fernandez-Lodeiro, J. L. Capelo, and C. Lodeiro, Sens. Actuators, B, 2015, 212, 297.

7. Y.-W. Lin, C.-C. Huang, and H.-T. Chang, Analyst, 2011, $136,863$.

8. E. Satheesh Kumar, J. Yang, V. Srinivasadesikan, and M.-C. Lin, Anal. Sci., 2017, 33, 1115.

9. Y.-F. Wang, N. Pan, and C.-F. Peng, Anal. Sci., 2017, 33, 321.

10. A. Kumar and R. K. Dutta, Anal. Sci., 2017, 33, 565.

11. Y. Zheng, X. Wang, and L. Xu, Anal. Sci., 2017, 33, 1345.

12. H. Iwai, Anal. Sci., 2017, 33, 1231.

13. S. Ito and M. Morita, Anal. Sci., 2016, 32, 1257.

14. J. Wu and N.-Y. Lee, Anal. Sci., 2016, 32, 85. 